\title{
ON THE CONSISTENCY AND EQUIVALENCE OF CERTAIN DEFINITIONS OF SUMMABILITY*
}

\author{
BY \\ WALLIE ABRAHAM HURWITZ AND L. L. SILVERMAN
}

\section{INTRODUCTION}

Many definitions have been proposed for the value of a divergent series, those of Cesàro and Hölder being familiar examples. All of the definitions proposed are generalizations of convergence; $\dagger$ that is, they evaluate any convergent series to the value to which it converges. Thus all these definitions give the same value to a convergent series. The fundamental question as to whether, when each of two definitions gives a value to a divergent series, the two values are the same, seems as yet to have received no attention. $\ddagger$ That two definitions, both generalizations of convergence, may give different values to the same divergent series, is seen by the following example. Let the sequence defining the series be $x_{n}=(-1)^{n+1} \log n(n=1,2, \ldots)$; and let the value of the sequence be defined in two different ways by the limits of the sequences $\left(y_{n}\right),\left(z_{n}\right)$, where

$$
y_{n}=\frac{1}{n} \sum_{k=1}^{n} x_{k}, \quad z_{n}=\frac{1}{n} \sum_{k=2}^{n}\left[1+\frac{(-1)^{k+1}}{\log k}\right] x_{k} \quad(n=2,3, \cdots) .
$$

It can easily be verified that each of the definitions is a generalization of convergence; whereas

$$
\lim _{n=\infty} y_{n}=0, \quad \lim _{n=\infty} z_{n}=1 .
$$

Furthermore the sequence $\left(x_{n}\right)$ may be made to give any preassigned value $\lambda$ whatever if we choose as the definition for the value of $\left(x_{n}\right)$ the limit of $s_{n}$, where $s_{n}=(1-\lambda) y_{n}+\lambda z_{n}$. It is accordingly a matter of the first importance to know under what circunstances two definitions are consistent; that is, under what circumstances we have a right to assert that whenever each of two definitions gives a value to a sequence, the two values are the same.

\footnotetext{
* Presented to the Society, September 8, 1914.

† Such definitions are sometimes said to satisfy the condition of consistency; the word consistency is used in this paper in a different sense.

$\ddagger$ Of course consistency is self-evident in the trivial case in which one definition evaluates all series evaluated by another definition, giving the same values.
} 
The principal result of this paper is that all definitions of summability of a certain class are consistent.

Another result of some interest is the determination of a criterion for the equivalence of two definitions of summability; two definitions being defined as equivalent when each evaluates to the value $\xi$ every sequence evaluated by the other to the value $\xi$. The interest in this idea seems hitherto to have been directed to the proof of the equivalence of Cesàro's and Hölder's definitions for the same order of summability, though other similar special questions have been considered. In this paper a criterion is established for the equivalence of any two definitions of a certain class, from which criterion the equivalence of Cesàro's and Hölder's definitions follows as a very special case.

Other points considered are: the specification of a definition which shall evaluate the sum of two given sequences summable by two stated definitions; the establishment of a necessary condition for summability, analogous to the well-known conditions for the cases of convergence and of Hölder-summability; and the permissibility of omitting or adjoining an element at the beginning of a summable sequence without altering either its summability or the value to which it is summable.

We shall be concerned with a special type* of definition of summability. Let $\left(x_{n}\right)=x_{1}, x_{2}, \cdots$ be a sequence, and

$$
\left(a_{n, k}\right)=\left\|\begin{array}{cccc}
a_{1,1}, & 0, & 0, & \cdots \\
a_{2,1}, & a_{2,2}, & 0, & \cdots \\
a_{3,1}, & a_{3,2}, & a_{3,3}, & \cdots \\
\vdots & \vdots & \vdots
\end{array}\right\|
$$

an infinite matrix of numbers, real or complex. Then the transformation

$$
y_{n}=\sum_{k=1}^{n} a_{n, k} x_{k} \quad(n=1,2, \cdots)
$$

defines the sequence $\left(y_{n}\right)=y_{1}, y_{2}, \ldots$. We indicate the transformation or the matrix by $A$ and the relation by $\left(y_{n}\right)=A\left(x_{n}\right)$. If

$$
\lim _{n=\infty} y_{n}=\xi,
$$

we define $\xi$ to be the value given to $\left(x_{n}\right)$ by the definition or transformation $A$. If $a_{n n} \neq 0(n=1,2, \cdots)$, we may solve for $x_{n}$,

$$
x_{n}=\sum_{k=1}^{n} \alpha_{n, k} y_{k} \quad(n=1,2, \cdots) ;
$$

* This type was first studied by Silverman, Missouri dissertation, 1910, and University of Missouri Studies, Mathematics Series, vol. 1, No. 1 (1913); Toeplitz, P r a c e m a t e m a tyczno fizyczno, vol. 22 (1911), p. 113; Smail, Columbia dissertation, 1913; and Schur, Mathematische Annalen, vol. 74 (1913), p. 447. 
we denote this transformation by $A^{-1}$ and the relation by $\left(x_{n}\right)=A^{-1}\left(y_{n}\right)$. If $\left(y_{n}\right)=A\left(x_{n}\right)$ and $z_{n}=B\left(y_{n}\right)$, then $z_{n}=B\left(A\left(x_{n}\right)\right)$, the transformation being $B A$. If $A B=B A, A$ and $B$ are permutable. If $A$ and $B$ correspond to $\left(a_{n, k}\right)$ and $\left(b_{n, k}\right)$ respectively, $\alpha A+\beta B$ will correspond to $\left(\alpha a_{n, k}+\beta b_{n, k}\right)$. If $A_{1}, A_{2}, \cdots$ correspond to $\left(a_{n, k}^{(1)}\right),\left(a_{n, k}^{(2)}\right), \cdots$ respectively, $\alpha_{1} A_{1}+\alpha_{2} A_{2}+\cdots$ will correspond to $\left(a_{n, k}\right)$ if

$$
\lim _{p=\infty}\left[\alpha_{1} a_{n, k}^{(1)}+\alpha_{2} a_{n, k}^{(2)}+\cdots+\alpha_{p} a_{n, k}^{(p)}\right]
$$

exists and equals $a_{n, k}$. If $\left(y_{n}\right)=A\left(x_{n}\right)$, and $\left(y_{n}\right)$ has the limit $\xi$ whenever $\left(x_{n}\right)$ has the limit $\xi, A$ is regular. It will be seen that if $A$ and $B$ are regular, then $A B$, and for any constant $\alpha, \alpha A+(1-\alpha) B$ are regular.

A necessary and sufficient condition that $A$ be regular* is
(a) $\lim _{n=\infty} a_{n, k}=0$,
(b) $\lim _{n=\infty} \sum_{k=1}^{n} a_{n, k}=1$,
(c) $\sum_{k=1}^{n}\left|a_{n, k}\right|<K$

The simplest examples of regular transformations are

and

$$
E: \quad a_{n, k}=0, \quad n \neq k ; \quad a_{n, n}=1 ;
$$

$$
M: a_{n, k}=\frac{1}{n} .
$$

Schur has studied the transformation $\alpha E+(1-\alpha) M$. It is natural to consider the more general transformation

$$
\alpha_{0} E+\alpha_{1} M+\alpha_{2} M^{2}+\cdots+\alpha_{n} M^{n},
$$

or still more generally the symbol

$$
\alpha_{0} E+\alpha_{1} M+\alpha_{2} M^{2}+\cdots,
$$

and to ask under what conditions it defines a regular transformation.

Theorem I. If $f(z)=\alpha_{0}+\alpha_{1} z+\alpha_{2} z^{2}+\cdots$ is analytic within and on the boundary of a circle of unit radius about the origin, and $f(1)=1$, then the symbol $\alpha_{0} E+\alpha_{1} M+\alpha_{2} M^{2}+\cdots$ gives rise to a regular transformation.

Let $E, M, M^{2}, \cdots$ correspond to matrices $m_{n, k}^{(0)}, m_{n, k}^{(1)}, m_{n, k}^{(2)}, \cdots$, respectively. Then we have

$$
\sum_{r=0}^{p}\left|\alpha_{r} m_{n, k}^{(r)}\right| \leqq \sum_{r=0}^{p}\left|\alpha_{r}\right|
$$

* The sufficiency of the condition was proved by Silverman and the necessity by Toeplitz in the papers previously cited. We shall use only the fact of sufficiency; indeed certain results obtained by Schur by means of the necessity of the condition are here proved without this part of the criterion. 
since $0 \leqq m_{n, k}^{(r)} \leqq 1$. Hence

$$
\lim _{p=\infty} \sum_{r=0}^{p} \alpha_{r} m_{n, k}^{(r)}
$$

exists uniformly in $k$; call its value $a_{n, k}$. Therefore*

$$
\lim _{n=\infty} a_{n, k}=\lim _{n=\infty} \lim _{p=\infty} \sum_{r=0}^{p} \alpha_{r} m_{n, k}^{(r)}=\lim _{p=\infty} \lim _{n=\infty} \sum_{r=0}^{p} \alpha_{r} m_{n, k}^{(r)}=0 .
$$

$$
\begin{aligned}
\sum_{k=1}^{n} a_{n, k} & =\sum_{k=1}^{n} \lim _{p=\infty} \sum_{r=0}^{p} \alpha_{r} m_{n, k}^{(r)}=\lim _{p=\infty} \sum_{r=0}^{p} \alpha_{r} \sum_{k=1}^{n} m_{n, k}^{(r)} \\
& =\lim _{p=\infty} \sum_{r=0}^{p} \alpha_{r}=f(1)=1 .
\end{aligned}
$$

$$
\sum_{k=1}^{n}\left|a_{n, k}\right| \leqq \sum_{r=0}^{\infty}\left|\alpha_{r}\right|
$$

since, from the hypothesis, the series for $f(z)$ converges absolutely for $z=1$. As the conditions (a), (b), (c) of (1) are satisfied, the theorem is proved.

CoRorlary. The numbers $a_{n, k}$ are given in terms of $f(z)$ by the formula

$$
a_{n, k}=\sum_{h=k}^{n}(-1)^{h-k} \frac{(n-1) !}{(n-h) !(h-k) !(k-1) !} f\left(\frac{1}{h}\right) .
$$

We prove this first when $f(z)=z^{r}$, so that the transformation defined is $M^{r}$. It is to be shown that

$$
m_{n, k}^{(r)}=\sum_{h=k}^{n}(-1)^{h-k} \frac{(n-1) !}{(n-h) !(h-k) !(k-1) !} \frac{1}{h^{r}} .
$$

Suppose this holds for any $r$; to see that it holds for $r+1$, we write

$$
\begin{aligned}
m_{n, k}^{(r+1)} & =\frac{1}{n} \sum_{q=k}^{n} m_{q, k}^{(r)} \\
& =\frac{1}{n} \sum_{q=k}^{n} \sum_{h=k}^{q}(-1)^{h-k}(q-h) !(h-k) !(k-1) ! \frac{1}{h^{r}} \\
& =\frac{1}{n} \sum_{h=k}^{n} \sum_{q=h}^{n}(-1)^{h-k} \frac{(q-1) !}{(q-h) !(h-k) !(k-1) !} \frac{1}{h^{r}} \\
& =\frac{1}{n} \sum_{h=k}^{n}(h-k) !(k-1) ! \frac{1}{h^{r}} \sum_{q=h}^{n}(q-1) ! \\
& =\sum_{h=k}^{n}(-1)^{h-k}(n-k) !
\end{aligned}
$$

* Osgood, Lehrbuch der Funktionentheorie, vol. 1, 2d ed., p. 593. 
As evidently (3) is true for $r=0$, it is true for all values of $r$. Finally, to prove (2) in general, multiply (3) by $\alpha_{r}$ and sum from $r=0$ to $r=\infty$. Then

$$
\begin{aligned}
\sum_{r=0}^{\infty} \alpha_{r} m_{n, k}^{(r)} & =\sum_{r=0}^{\infty} \sum_{h=k}^{n}(-1)^{h-k} \frac{(n-1) !}{(n-h) !(h-k) !(k-1) !} \frac{\alpha_{r}}{h^{r}} \\
& =\sum_{h=k}^{n}(-1)^{h-k} \frac{(n-1) !}{(n-h) !(h-k) !(k-1) !} \sum_{r=0}^{\infty} \frac{\alpha_{r}}{h^{r}},
\end{aligned}
$$

which agrees with (2).

\section{Consistency of transformations PERmutable with $M$}

We shall now determine a sufficient condition for the consistency of two regular definitions of summability. We shall refer to any transformation of the form

$$
y_{n}=f_{n} x_{n}
$$

as a multiplication. We have occasion to use Euler's transformation*

$$
y_{n}=\sum_{k=1}^{n}(-1)^{k-1} \frac{(n-1) !}{(n-k) !(k-1) !} x_{k},
$$

which we shall call $\Delta$. It satisfies the condition

$$
\Delta^{2}=E,
$$

as may be seen by actual repetition of (5) or more easily by solving the expanded formulae:

$$
\begin{aligned}
& y_{1}=x_{1}, \\
& y_{2}=x_{1}-x_{2}, \\
& y_{3}=x_{1}-2 x_{2}+x_{3},
\end{aligned}
$$

Lemma 1. If $M^{\prime}$ denotes the multiplication

$$
y_{n}=\frac{1}{n} x_{n},
$$

then

$$
M=\Delta M^{\prime} \Delta, \quad M^{\prime}=\Delta M \Delta .
$$

To prove this, form $M \Delta$; we have

$$
y_{n}=\frac{1}{n} \sum_{k=1}^{n} \sum_{h=1}^{k}(-1)^{h-1} \frac{(k-1) !}{(k-h) !(h-1) !} x_{h}
$$

* Bromwich, An Introduction to the Theory of Infinite Series, p. 303. 


$$
\begin{aligned}
& =\frac{1}{n} \sum_{h=1}^{n}(-1)^{h-1} x_{h}\left(\sum_{k=h}^{n} \frac{(k-1) !}{(k-h) !(h-1) !}\right) \\
& =\frac{1}{n} \sum_{h=1}^{n}(-1)^{h-1} \frac{n !}{(n-h) ! h !} x_{h} \\
& =\sum_{h=1}^{n}(-1)^{h-1} \frac{(n-1) !}{(n-h) !(h-1) !}\left(\frac{x_{h}}{h}\right) .
\end{aligned}
$$

But this is obviously the result of applying $\Delta$ to the sequence $\left(x_{n} / n\right)$, - that is, to the result of transforming $\left(x_{n}\right)$ by $M^{\prime}$. Hence

$$
M \Delta=\Delta M^{\prime}
$$

and the proposition follows at once by (6).

LEMma 2. A necessary and sufficient condition that $A$ be permutable with $M$ is that $A^{\prime}$ be permutable with $M^{\prime}$, where $A^{\prime}=\Delta A \Delta$.

If $A M=M A$, then

$A^{\prime} M^{\prime}=(\Delta A \Delta)(\Delta M \Delta)=\Delta(A M) \Delta=\Delta(M A) \Delta$

Conversely, let $A^{\prime} M^{\prime}=M^{\prime} A^{\prime}$; then

$$
=(\Delta M \Delta)(\Delta A \Delta)=M^{\prime} A^{\prime} .
$$

$$
\begin{aligned}
A M=\left(\Delta A^{\prime} \Delta\right)\left(\Delta M^{\prime} \Delta\right)=\Delta\left(A^{\prime} M^{\prime}\right) \Delta=\Delta\left(M^{\prime} A^{\prime}\right) \Delta \\
=\left(\Delta M^{\prime} \Delta\right)\left(\Delta A^{\prime} \Delta\right)=M A .
\end{aligned}
$$

Lemma 3. A necessary and sufficient condition that a transformation be permutable with $M^{\prime}$ is that it be a multiplication.

Suppose the transformation $A^{\prime}$,

$$
y_{n}=\sum_{k=1}^{n} a_{n, k} x_{k}
$$

is permutable with $M^{\prime}$. Then writing $M^{\prime} A^{\prime}=A^{\prime} M^{\prime}$, we have

Hence

$$
\frac{1}{n} \sum_{k=1}^{n} a_{n, k} x_{k}=\sum_{k=1}^{n} a_{n, k} \frac{x_{k}}{k}
$$

$$
\frac{a_{n, k}}{n}=\frac{a_{n, k}}{k}
$$

or

$$
(n-k) a_{n, k}=0 \text {. }
$$

Therefore, when $k \neq n, a_{n, k}=0$. Writing $a_{n, n}=f_{n}$, we have the multiplication (4).

Conversely, if we denote by $A^{\prime}$ the transformation (4), obviously

$$
M^{\prime} A^{\prime}=A^{\prime} M^{\prime} \text {. }
$$

Combining the two preceding lemmas, we have 
Lemma 4. A necessary and sufficient condition that $A$ be permutable with $M$ is that there exist a multiplication $A^{\prime}$ such that $A=\Delta A^{\prime} \Delta$.

We shall now say that $A$ corresponds to $A^{\prime}$ if $A=\Delta A^{\prime} \Delta$.

Lemma 5. If $A$ and $B$ correspond to $A^{\prime}$ and $B^{\prime}$ respectively, then $A B$ corresponds to $A^{\prime} B^{\prime}$.

For $A B=\left(\Delta A^{\prime} \Delta\right)\left(\Delta B^{\prime} \Delta\right)=\Delta\left(A^{\prime} B^{\prime}\right) \Delta$.

Lemma 6. Two transformations, each permutable with $M$, are permutable with each other.

For two multiplications are obviously permutable; hence if $A, B$ correspond respectively to $A^{\prime}, B^{\prime}$, then $A B$ and $B A$, which correspond by Lemma 5 to $A^{\prime} B^{\prime}$ and $B^{\prime} A^{\prime}$, are equal.

THEOREM II. All regular definitions permutable with $M$ are consistent.

Let $A$ and $B$ be any two regular definitions permutable with $M$, evaluating the sequence $\left(x_{n}\right)$ to $\xi$ and $\eta$ respectively. Then $\left(x_{n}\right)$ is evaluated by $B A$ to $\xi$ and by $A B$ to $\eta$. Since by Lemma $6, A B=B A$, it follows that $\xi=\eta$.

Theorem III. If $A, B$ are regular definitions permutable with $M$, and if $A$ evaluates $\left(x_{n}\right)$ to $\xi$ and $B$ evaluates $\left(y_{n}\right)$ to $\eta$, then $A B$ evaluates $\left(x_{n}+y_{n}\right)$ to $\xi+\eta$.

For evidently $A B$ evaluates $\left(x_{n}\right)$ to $\xi$ and $\left(y_{n}\right)$ to $\eta$.

Theorem IV. $A$ necessary and sufficient condition that $A$ be permutable with $M$ is that there exist numbers $f_{1}, f_{2}, \ldots$ such that.

$$
a_{n, k}=\sum_{h=k}^{n}(-1)^{h-k} \frac{(n-1) !}{(n-h) !(h-k) !(k-1) !} f_{h} .
$$

If $A$ is permutable with $M$, then by Lemma $4, A=\Delta A^{\prime} \Delta$, where $A^{\prime}$ is given by (4). Hence we have for $A$ the formula

$$
\begin{aligned}
y_{n}= & \sum_{h=1}^{n}(-1)^{h-1} \frac{(n-1) !}{(n-h) !(h-1) !} f_{h} \\
& \times\left(\sum_{k=1}^{h}(-1)^{k-1} \frac{(h-1) !}{(h-k) !(k-1) !} x_{k}\right) \\
& =\sum_{k=1}^{n}\left(\sum_{h=k}^{n}(-1)^{h-k} \frac{(n-1) !}{(n-h) !(h-k) !(k-1) !} f_{h}\right) x_{k},
\end{aligned}
$$

which proves (9).

Conversely, if (9) is satisfied, (10) shows that $A=\Delta A^{\prime} \Delta$, where $A^{\prime}$ is a multiplication.

\section{Regularity of transformations permutable With $M$}

We have seen that the symbol $\alpha_{0} E+\alpha_{1} M+\alpha_{2} M^{2}+\cdots$ gives rise to a regular transformation if $f(z)=\alpha_{0}+\alpha_{1} z+\alpha_{2} z^{2}+\cdots$ is analytic in the 
unit circle. We consider next a general function $f(z)$ of the complex variable $z$; and we define $f(M)$, the corresponding function of $M$, to be a transformation for which the numbers $a_{n, k}$ are given by the formula*

$$
a_{n, k}=\sum_{h=k}^{n}(-1)^{h-k} \frac{(n-1) !}{(n-h) !(h-k) !(k-1) !} f\left(\frac{1}{h}\right) .
$$

It is evident from Theorem IV of the preceding section that $f(M)$ is permutable with $M$, and from Lemma 5, that if $h(z)=f(z) g(z)$, then $h(M)$ $=f(M) g(M)$. We shall now prove a theorem which contains Theorem I as a special case.

THEOREM V. The transformation $f(M)$ is regular if $f(z)$ is analytic within and on the boundary of the circle $C$ of radius $\frac{1}{2}$ about the point $\frac{1}{2}$, and $f(1)=1$.

By hypothesis $f(z)$ is analytic in a circle $C_{e}$ of radius $\frac{1}{2}+\epsilon$ about the point $\frac{1}{2}$, where $\epsilon$ is a sufficiently small positive number. Since all the points $1, \frac{1}{2}, \frac{1}{3}, \cdots$ lie inside $C_{e}$, we have by Cauchy's integral-theorem

$$
f\left(\frac{1}{h}\right)=\frac{1}{2 \pi i} \int_{C_{\epsilon}} \frac{f(t)}{t-\frac{1}{h}} d t .
$$

Substituting this value in (11),

$$
\begin{aligned}
a_{n, k} & =\frac{1}{2 \pi i} \sum_{h=k}^{n}(-1)^{h-k} \frac{(n-1) !}{(n-h) !(h-k) !(k-1) !} \int_{C_{\mathrm{e}}} \frac{f(t)}{t-\frac{1}{h}} d t \\
& =\frac{1}{2 \pi i} \int_{C_{\mathrm{e}}} \sum_{h=k}^{n}(-1)^{h-k} \frac{(n-1) !}{(n-h) !(h-k) !(k-1) !} \frac{f(t)}{t-\frac{1}{h}} d t .
\end{aligned}
$$

But $\dagger$

$$
\sum_{h=k}^{n}(-1)^{h-k} \frac{n !}{(n-h) !(h-k) !(k-1) !} \frac{1}{t-\frac{1}{h}}=t^{n-k-1} \prod_{h=k}^{n} \frac{1}{t-\frac{1}{h}}, \quad n>k .
$$

so that

$$
\begin{aligned}
a_{n, k} & =\frac{1}{2 \pi i n} \int_{C_{e}} f(t) t^{n-k-1} \prod_{h=k}^{n} \frac{1}{t-\frac{1}{h}} d t \\
& =\frac{1}{2 \pi i n} \int_{C_{e}} \frac{f(t)}{t^{2}} \prod_{h=k}^{n} \frac{1}{1-\frac{1}{h t}} d t, \quad n>k .
\end{aligned}
$$

* This formula is identical with (2) of Section 1.

† This may be proved at once by resolving the right-händ side into partial fraction. 
Now $C_{\epsilon}$ is a circle whose diameter joins the points $-\epsilon$ and $1+\epsilon$. Thus when $t$ lies on $C_{e}, h t$ lies on a circle whose diameter joins the points $-h \epsilon$ and $h(1+\epsilon)$, and $1 / h t$ lies on a circle whose diameter joins the points $-1 / h \epsilon$ and $1 / h(1+\epsilon)$; hence

$$
\left|1-\frac{1}{h t}\right| \geqq 1-\frac{1}{h(1+\epsilon)} .
$$

Let $L$ be a constant greater than the absolute value, on $C_{e}$, of $f(t) / t^{2}$, and let $\delta=1 /(1+\epsilon)$, and $N=L\left(\frac{1}{2}+\epsilon\right)$. Then

$$
\left|a_{n, k}\right| \leqq \frac{L}{2 \pi n} \int_{C_{\mathrm{e}}, \prod_{h=k}^{n}} \frac{1}{1-\frac{\delta}{h}} d s=\frac{N}{n} \prod_{h=k}^{n} \frac{h}{h-\delta}, \quad n>k .
$$

To prove that $f(M)$ is regular we shall show that the conditions (1) are fulfilled.

(a) We find

$$
\begin{aligned}
\left|a_{n, k}\right| & \leqq \frac{N}{n} \prod_{h=k}^{n} \frac{h}{h-\delta} \\
& =\frac{(n-k)^{k-\delta} \frac{1 \cdot 2 \cdot \cdots \cdot(n-k)}{(k-\delta)(k+1-\delta) \cdots(n-\delta)}}{(n-k)^{k} \frac{1 \cdot 2 \cdot \cdots \cdot(n-k)}{k(k+1) \cdot \cdots \cdot n}} N\left(\frac{n-k}{n}\right)^{\delta} \frac{1}{n^{1-\delta}} .
\end{aligned}
$$

Hence

$$
\lim _{n=\infty}\left|a_{n, k}\right|=\frac{\Gamma(k-\delta)}{\Gamma(k)} \cdot N \cdot 1 \cdot 0=0
$$

(b) We have

$$
\begin{aligned}
\sum_{k=1}^{n} a_{n, k} & =\sum_{k=1}^{n} \sum_{h=k}^{n}(-1)^{h-k} \frac{(n-1) !}{(n-h) !(h-k) !(k-1) !} f\left(\frac{1}{h}\right) \\
& =\sum_{h=1}^{n} \sum_{k=1}^{n}(-1)^{h-k} \frac{(n-1) !}{(n-h) !(h-k) !(k-1) !} f\left(\frac{1}{h}\right) .
\end{aligned}
$$

Now the coefficient of $f(1 / h)$ may be written

$$
\frac{(n-1) !}{(n-h) !(h-1) !} \sum_{k=1}^{h}(-1)^{h-k} \frac{(h-1) !}{(h-k) !(k-1) !},
$$

which equals* unity for $h=1$ and zero for all other values of $h$. Hence

$$
\sum_{k=1}^{n} a_{n, k}=f(1)=1
$$

* The summation is obviously equal to the sum of the coefficients in the expansion of $(-1+x)^{h-1}$. 
(c) We first find

$$
\begin{aligned}
\left|a_{n, 1}\right|+\left|a_{n, 2}\right| & \leqq \frac{N}{n} \prod_{h=1}^{n} \frac{h}{h-\delta}+\frac{N}{n} \prod_{h=2}^{n} \frac{h}{h-\delta} \bar{h} \\
& =\frac{N}{n} \frac{2}{1-\delta} \prod_{h=3}^{n} \frac{h}{h-\delta},
\end{aligned}
$$

and then, by mathematical induction,

so that

$$
\sum_{k=1}^{p \prime}\left|a_{n, k}\right| \leqq \frac{N p}{n(1-\delta)} \prod_{h=p+1}^{n} \frac{h}{h-\delta} \quad(p<n),
$$

$$
\sum_{k=1}^{n-1}\left|a_{n, k}\right| \leqq \frac{N(n-1)}{n(1-\delta)}
$$

Since the limit, as $n$ becomes infinite, of the expression on the right exists, it follows that there exists a positive constant $H$ such that

$$
\sum_{k=1}^{n-1}\left|a_{n, k}\right|<H
$$

Furthermore, since $a_{n, n}=f(1 / n)$, there exists a positive constant $J$ such that Hence

$$
\left|a_{n, n}\right|<J \text {. }
$$

$$
\sum_{k=1}^{n}\left|a_{n, k}\right|<H+J
$$

Corollary. The Hölder and Cesàro means of order $r$ are respectively $f(M)$ and $g(M)$, where

$$
\begin{gathered}
f(z)=z^{r} \\
g(z)=\frac{r ! z^{r}}{(1+z)(1+2 z) \cdots(1+\overline{r-1} z)} .
\end{gathered}
$$

The proof is immediate. It may at once be verified that the Hölder and Cesàro means are permutable with $M$; it is therefore sufficient to give for each case a function analytic in $C$, having the value 1 for $z=1$ and the value $a_{n, n}$ for $1 / n$. For the Hölder mean of order $r, a_{n, n}=1 / n^{r}$; hence the transformation is defined by $f(z)=z^{r}$. For the Cesàro mean of order $r$,

$$
\begin{aligned}
a_{n, n}=\frac{r !}{(r+n-1)(r+n-2)} & \cdots n \\
= & \frac{r !}{n^{r}\left(1+\frac{1}{n}\right)\left(1+\frac{2}{n}\right) \cdots\left(1+\frac{r-1}{n}\right)} ;
\end{aligned}
$$

so that the transformation is defined by the function $g(z)$ given above. 


\section{A CASE OF IRREgULARITY}

As a case of irregularity it will be of value to study the effect of poles of the function $f(z)$. The simplest function possessing a pole of the first order and having the value 1 at $z=1$ is $f(z)=(1-\rho) /(z-\rho)$, where $\rho \neq 1$.

LEMMA 1: The function

$$
f(z)=\frac{1-\rho}{z-\rho}
$$

where $\rho \neq 1$ is a point within or on the boundary of the circle $C$, does not define a regular transformation.

Disregarding the cases in which $\rho$ is the reciprocal of a positive integer, since in those cases the formula (11) for the coefficients of the matrix corresponding to $f(z)$ breaks down, and excluding the case $\rho=0$, since in that case $f(z)$ defines the transformation $M^{-1}$, which is obviously not regular, we proceed to set up a sequence $\left(x_{n}\right)$ and the transformed sequence $\left(y_{n}\right)$ in such a way that the former has the limit zero, while the latter does not. It will be simpler to define $\left(y_{n}\right)$ first. We may then find $\left(x_{n}\right)$ by performing the transformation corresponding to $1 / f(z)=(z-\rho) /(1-\rho)$, so that

$$
(1-\rho) x_{n}=\frac{1}{n} \sum_{h=1}^{n} y_{h}-\rho y_{n} .
$$

We take

$$
y_{n}=\frac{\Gamma(n)}{\Gamma(n-p)}, \quad p=\frac{1}{\rho}-1
$$

The restrictions on $\rho$ show that $\mathbf{R}(p) \geqq 0$ and that $p \neq 0,1,2, \ldots$. From these two facts we see that $y_{n}$ is defined for $n=1,2, \cdots$; and that*

$$
\begin{array}{cll}
\lim _{n=\infty} y_{n}=\infty, & \text { if } & \mathbf{R}(p)>0 ; \\
\lim _{n=\infty}\left|y_{n}\right|=1, & \text { if } & \mathbf{R}(p)=0 ;
\end{array}
$$

so that in neither case does $y_{n}$ approach zero.

On the other hand we find

$$
\frac{\Gamma(n+1)}{\Gamma(n-p)}-\frac{\Gamma(n)}{\Gamma(n-p-1)}=(p+1) \frac{\Gamma(n)}{\Gamma(n-p)},
$$

so that

$$
y_{n}=\rho \frac{\Gamma(n+1)}{\Gamma(n-p)}-\rho \frac{\Gamma(n)}{\Gamma(n-p-1)},
$$

* The ratio $\Gamma(n) / \Gamma(n-p)$ is readily studied by Stirling's Theorem. It is seen that the limit is 0 or $\infty$ accurding as $\mathbf{R}(p)<$ or $>0$; if $\mathbf{R}(p)=0$, the absolute value of the ratio has the limit 1 . 
and

$$
\sum_{h=1}^{n} y_{h}=\rho \frac{\Gamma(n+1)}{\Gamma(n-p)}-\rho \frac{1}{\Gamma(-p)}
$$

Denoting by $c$ the expression $\rho / \Gamma(-p)$, which is independent of $n$, we have from (14), (15), and (16),

so that

$$
(1-\rho) x_{n}=\rho \frac{\Gamma(n)}{\Gamma(n-p)}-\frac{c}{n}-\rho \frac{\Gamma(n)}{\Gamma(n-p)}=-\frac{c}{n},
$$

$$
\lim _{n=\infty} x_{n}=0 \text {. }
$$

LemMa 2. If $f(z)$ is analytic within and on the boundary of the circle $C$ except for a single pole of the first order, and $f(1)=1, f(z)$ does not define a regular transformation.

If $\rho$ is the pole, we must be able to write

$$
f(z)=a \frac{1-\rho}{z-\rho}+g(\dot{s}), \quad a \neq 0,
$$

where $g(z)$ is analytic throughout $C$. Assuming first $g(1) \neq 0$, we have from (17)

$$
\begin{gathered}
\frac{1-\rho}{z-\rho}=\frac{1}{a} f(z)-\frac{1}{a} g(z) \\
=\frac{1}{a} f(z)-\frac{g(1)}{a} \frac{g(z)}{g(1)} .
\end{gathered}
$$

Suppose now that $f(z)$ does define a regular transformation. Then calling $A$ and $B$ the regular transformations defined by $f(z)$ and $g(z) / g(1)$ respectively, we should find that the transformation

$$
\frac{1}{a} A-\frac{g(1)}{a} B
$$

is regular, since* from the assumption $f(1)=1$ and from (18),

$$
\frac{1}{a}-\frac{g(1)}{a}=1 \text {. }
$$

Hence it would follow from (18) that the function $(1-\rho) /(z-\rho)$ defines a regular transformation, in contradiction to the preceding lemma.

In the case $g(1)=0$, it follows from (17) that $a=1$; and (18) becomes

$$
\frac{1-\rho}{z-\rho}=f(z)-g(z),
$$

* See p. 3. 
so that

$$
\frac{1}{2}\left(1+\frac{1-\rho}{z-\rho}\right)=\frac{1}{2} f(z)+\frac{1}{2}[1-g(z)]
$$

If it is now assumed that $f(z)$ defines a regular transformation it will follow, since $1-g(z)$ defines a regular transformation, that

$$
\phi(z)=\frac{1}{2}\left(1+\frac{1-\rho}{z-\rho}\right)
$$

and hence that

$$
\frac{1-\rho}{z-\rho}=2 \phi(z)-1
$$

defines a regular transformation. But this again contradicts the preceding lemma.

Theorem VI. If $f(z)$ has at least one pole, but is analytic except for poles within and on the boundary of the circle $C$, and $f(1)=1$, then $f(z)$ does not define a regular transformation.

The number of poles must be finite; denote them, each repeated as often as its multiplicity indicates, by $\rho_{1}, \rho_{2}, \cdots, \rho_{n}$. Then

$$
f(z)=\frac{1-\rho_{1}}{z-\rho_{1}} \cdot \frac{1-\rho_{2}}{z-\rho_{2}} \cdots \frac{1-\rho_{n}}{z-\rho_{n}} g(z),
$$

where $g(z)$ is analytic throughout $C$. If $f(z)$ defines a regular transformation, so will the product of $f(z)$ by the analytic function

$$
\frac{z-\rho_{2}}{1-\rho_{2}} \cdot \frac{z-\rho_{3}}{1-\rho_{3}} \cdots \cdot \frac{z-\rho_{n}}{1-\rho_{n}}
$$

this product, however, possesses a single pole $\rho_{1}$ of the first order, and by Lemma 2 does not define a regular transformation.

\section{ANalytically REgUlar transformations}

We shall now use the term analytically regular to describe a transformation $f(M)$ defined by a function $f(z)$ analytic throughout $C$, having the value 1 for $z=1$. A number of properties of such transformations follow immediately from the results of the two preceding sections.

Theorem VII. All analytically regular definitions are consistent.

This is evident by Theorem II, since all these transformations, being of the form (9), are, by Theorem IV, permutable with $M$.

Theorem VIII. If $f(M), g(M)$ are analytically regular transformations, a necessary and sufficient condition that $f(M)$ should evaluate every sequence 
which $g(M)$ evaluates, giving it the same value, is that all the zeros of $g(z)$ in $C$ should be zeros of at least as high order of $f(z)$.

If $\left(x_{n}\right)$ be any sequence transformed by $f(M)$ to $\left(u_{n}\right)$ and by $g(M)$ to $\left(v_{n}\right)$, then $\left(v_{n}\right)$ is transformed to $\left(u_{n}\right)$ by $h(M)$, where $h(z)=f(z) / g(z)$. In order that the condition of the theorem be satisfied, it is necessary and sufficient that $h(M)$ be regular. As the only possible singularities of $h(z)$ are poles due to the zeros of $g(z), h(M)$ will be regular if there are no poles (by $\S 3$ ), that is, if the zeros of $g(z)$ are zeros of the same or higher orders of $f(z)$; and will not be regular in the contrary case (by $\S 4$ ).

As an immediate deduction we have the two following theorems.

TheoRem IX. If $f(M), g(M)$ are analytically regular, a necessary and sufficient condition that they be equivalent is that $f(z), g(z)$ have in $C$ the same zeros with the same orders.

CoROLlary. The Hölder and Cesàro means of like order are equivalent.

For the functions $f(z), g(z)$ of (11), (12) are analytic in $C$; each has no zeros except $z=0$, and this is in both cases a zero of order $r$; hence the two definitions are equivalent.

Theorem X. A necessary and sufficient condition that the analytically regular definition $f(M)$ be reversible (equivalent to convergence) is that $f(z)$ do not vanish in $C$.

\section{A NECESSARY CONDITION FOR SUMMABILITY}

In the cases of convergence and of Cesàro and Hölder summability, there exists a simple form of necessary condition, applied usually to the general term of the infinite series, that is, to the difference of two elements of the sequence. We have a similar test in the case of a wide range of definitions of the type which we are considering.

Theorem XI. If the sequence $\left(x_{n}\right)$ is transformed into a convergent sequence by the analytically regular transformation $f(M)$ defined by a function $f(z)$ which has no zeros within or on the boundary of the circle $C^{\prime}$ of radius $\frac{1}{4}$ about the point $\frac{1}{4}$, then

$$
\lim _{n=\infty}\left(x_{n}-x_{n-1}\right)=0 \text {. }
$$

We suppose that the function $f(z)$ defines the transformation $f(M)$ which has as coefficients of its matrix $\left(f_{n, k}\right)$, and that similarly $g(z), a(z), b(z)$ define respectively the transformations $g(M), a(M), b(M)$, with coefficients $\left(g_{n, k}\right),\left(a_{n, k}\right),\left(b_{n, k}\right)$, where

$$
g(z)=\frac{1}{f(z)}, \quad a(z)=\frac{g\left(\frac{z}{z+1}\right)}{g\left(\frac{1}{2}\right)}, \quad b(z)=\frac{1}{z} a(z) .
$$


Since $f(z)$ has no zeros in $C^{\prime}, f[z /(z+1)]$ has no zeros in $C$, hence $a(z)$ is analytic in $C$ and $a(M)$ is analytically regular. Now let

$$
y_{n}=\sum_{k=1}^{n} f_{n, k} x_{k}
$$

then

$$
x_{n}=\sum_{k=1}^{n} g_{n, k} y_{k},
$$

so that

$$
x_{n}-x_{n-1}=g_{n, n} y_{n}+\sum_{k=1}^{n-1}\left(g_{n, k}-g_{n-1, k}\right) y_{k}
$$

From

$$
g_{n, k}=\sum_{h=k}^{n}(-1)^{h-k} \frac{(n-1) !}{(n-h) !(h-k) !(k-1) !} g\left(\frac{1}{h}\right)
$$

we find

$$
\begin{aligned}
g_{n, k}-g_{n-1, k} & =\frac{g\left(\frac{1}{2}\right)}{k-1} b_{n-1, k-1} \\
g_{n, 1}-g_{n-1,1} & =-g\left(\frac{1}{2}\right) a_{n-1,1} \\
g_{n, n} & =\frac{g\left(\frac{1}{2}\right)}{n-1} b_{n-1, n-1} .
\end{aligned}
$$

Hence

$$
\begin{aligned}
x_{n}-x_{n-1} & =-g\left(\frac{1}{2}\right) a_{n-1,1} y_{1}+g\left(\frac{1}{2}\right) \sum_{k=2}^{n} b_{,-1, k-1} \frac{y_{k}}{k-1} \\
& =-g\left(\frac{1}{2}\right) a_{n-1,1} y_{1}+g\left(\frac{1}{2}\right) \sum_{k=1}^{n-1} b_{n-1, k} \frac{y_{k+1}}{k}
\end{aligned}
$$

The expression given by the summation sign is the result of applying the transformation $b(M)$ to the sequence

$$
y_{2}, \frac{y_{3}}{2}, \frac{y_{4}}{3}, \cdots
$$

Since $b(z)=a(z) / z$, it suffices to apply to this sequence the transformation $M^{-1}$, and to the result the transformation $a(M)$. Hence the expression given by the summation is equal to

where

$$
\sum_{k=1}^{n-1} a_{n-1, k} v_{k},
$$

$$
v_{n-1}=(n-1) \frac{y_{n}}{n-1}-(n-2) \frac{y_{n-1}}{n-2}=y_{n}-y_{n-1}
$$


That is,

$$
\begin{aligned}
x_{n}-x_{n-1}= & -g\left(\frac{1}{2}\right) a_{n-1,1} y_{1}+g\left(\frac{1}{2}\right) \sum_{k=1}^{n-1} a_{n-1, k}\left(y_{k+1}-y_{k}\right) \\
= & g\left(\frac{1}{2}\right)\left[a_{n-1,1}\left(y_{2}-2 y_{1}\right)+a_{n-1,2}\left(y_{3}-y_{2}\right)+\cdots\right. \\
& \left.\quad+a_{n-1, n-1}\left(y_{n}-y_{n-1}\right)\right] .
\end{aligned}
$$

As the transformation $a(M)$ is analytically regular, and as, on account of the convergence of $\left(y_{n}\right)$,

it follows that

$$
\lim _{n=\infty}\left(y_{n}-y_{n-1}\right)=0 \text {, }
$$

as we wished to prove.

$$
\lim _{n=\infty}\left(x_{i,}-x_{n-1}\right)=0,
$$

COROLLARY. Under the same hypotheses as in the preceding theorem

$$
\lim _{n=\infty} \frac{x_{n}}{n}=0
$$

As proof we have only to consider the Hölder means of the sequence $x_{1}$, $x_{2}-x_{1}, x_{3}-x_{2}, \cdots$.

We obtain a more general theorem by taking a function $f(z)$ which has a zero of order $r$ at $z=0$, but no other zeros in $C^{\prime}$. Using the same notation as before, we have again (19). In the present case $z^{r} a(z)$ defines an analytically regular transformation; since the limit of the sequence $\left(y_{n}-y_{n-1}\right)$ is zero, the result of applying to this sequence first $a(M)$, then $M^{r}$, must give a sequence whose limit is zero; therefore the sequence $x_{n}-x_{n-1}$ is evaluated to zero by $M^{r}$. The usual test for Hölder summability gives

Thus we have

$$
\lim _{n=\infty} \frac{x_{n}-x_{n-1}}{n^{r}}=0 \text {. }
$$

Theorem XII. If a sequence $\left(x_{n}\right)$ is transformed into a convergent sequence by the analytically regular transformation $f(M)$ defined by a function $f(z)$ which has, except at $z=0$, no zeros within or on the boundary of the circle $C^{\prime}$ of radius $\frac{1}{4}$ about the point $\frac{1}{4}$, then

$$
\lim _{n=\infty} f\left(\frac{1}{n}\right)\left(x_{n}-x_{n-1}\right)=0 \text {. }
$$

\section{Omission and adjunction of elements at the beginNing of a SEQUENCE}

It is natural to ask under what circumstances the evaluability of the sequence $x_{1}, x_{2}, x_{3}, \cdots$ by a definition insures the evaluability of the srituence $x_{2}, x_{3}$, 
$x_{4}, \cdots$ by the same definition to the same value, and conversely. Two remarks should be made regarding this problem. In the first place, it is essentially equivalent to that of omitting or adjoining a term at the beginning of a series. To the series $u_{1}+u_{2}+u_{3}+\cdots$ corresponds the sequence $x_{1}=u_{1}$, $x_{2}=u_{1}+u_{2}, x_{3}=u_{1}+u_{2}+u_{3}, \cdots$; to the series $u_{2}+u_{3}+u_{4}+\cdots$ corresponds the sequence $x_{2}-u_{1}, x_{3}-u_{1}, x_{4}-u_{1}, \cdots$, which differs, element by element, from the sequence $x_{2}, x_{3}, x_{4}, \cdots$, only by the convergent sequence $u_{1}, u_{1}, u_{1}, \cdots$. Secondly, it is clear that in the case of any regular definition the possibility of adjoining an element is independent of the value of the element adjoined; it is the mere fact of alteration in rank of the elements which affects the summability.

Lemma. If, by the rth Cesàro mean, the sequence $x_{1}, x_{2}, x_{3}, \cdots$ is transformed into $y_{1}, y_{2}, y_{3}, \cdots$, and the sequence $x_{2}, x_{3}, x_{4}, \cdots$ into $\eta_{1}, \eta_{2}, \eta_{3}, \cdots$, then

$$
\begin{gathered}
y_{n}-\eta_{n}=\frac{r}{n} x_{1}-\frac{n+r}{n}\left(y_{n+1}-y_{n}\right)-\frac{r}{n} y_{n} \\
y_{n}-\eta_{n}=\frac{r}{n+r-1} x_{1}-\frac{n-1}{n+r-1}\left(\eta_{n}-\eta_{n-1}\right)-\frac{r}{n+r-1} \eta_{n} .
\end{gathered}
$$

From the formula for the Cesàro mean, we have

$$
\begin{aligned}
& y_{n}=r \frac{(n-1) !}{(n+r-1) !} \sum_{k=1}^{n} \frac{(n+r-k-1) !}{(n-k) !} x_{k}, \\
& \eta_{n-1}=r \frac{(n-2) !}{(n+r-2) !} \sum_{k=2}^{n} \frac{(n+r-k-1) !}{(n-k) !} x_{k}
\end{aligned}
$$

from these formulae follows at once

$$
(n+r-1) y_{n}-(n-1) \eta_{n-1}=r x_{1} .
$$

Solving for $y_{n}$, we obtain easily the second of the results to be proved; solving for $\eta_{n-1}$ and replacing $n$ by $n+1$, we obtain the first.

THEOREM XIII. If a sequence $\left(x_{n}\right)$ is transformed into a convergent sequence by the analytically regular transformation $f(M)$ defined by a function $f(z)$ which has, except at $z=0$, no zeros within or on the boundary of the circle $C^{\prime}$, then the sequence obtained by omitting or adjoining an element at the beginning is transformed by $f(M)$ into a sequence converging to the same value.

Suppose that $f(z)$ has at $z=0$ a zero of order $r$, and write*

$$
f(z)=\frac{r ! z^{r}}{(1+z)(1+2 z) \cdots(1+\overline{r-1} z)} g(z) ;
$$

* The factor multiplied into $g(z)$ is exactly the function which defines the $r$ th Cesaro mean given by (12). 
then $g(z)$ is analytic in $C$ and has no zeros in $C^{\prime}$. Using the notation of the preceding lemma, it suffices to show that if either of the sequences $\left(y_{n}\right)$, $\left(\eta_{n}\right)$ can be evaluated by $g(M)$, the other will be evaluated to the same value. This will be shown by proving the stronger statement, that if either $\left(y_{n}\right)$ or $\left(\eta_{n}\right)$ is evaluable by $g(M)$, then

$$
\lim _{n=\infty}\left(y_{n}-\eta_{n}\right)=0 \text {. }
$$

If $\left(y_{n}\right)$ is evaluable by $g(M)$, then by Theorem XI and its Corollary,

$$
\lim _{n=\infty}\left(y_{n+1}-y_{n}\right)=0, \quad \lim _{n=\infty} \frac{y_{n}}{n}=0 ;
$$

hence by (20) the assertion follows. Similarly if $\left(\eta_{n}\right)$ is evaluable by $g(M)$,

$$
\lim _{n=\infty}\left(\eta_{n+1}-\eta_{n}\right)=0, \quad \lim _{n=\infty} \eta_{n}=0,
$$

which by (21) yields the result.

It will finally be shown that the condition on $f(z)$ is essentially necessary,more accurately, that for any $\rho \neq 0$ in $C^{\prime}$ it is possible to construct a function $f(z)$ vanishing at $\rho$, and a sequence evaluable by $f(M)$ for which the dropping or adjoining an element is not permissible.

TheOREM XIV. If $f(z)=(z-\rho) /(1-\rho)$, where $\rho \neq 0$ is within or on the bourdary of $C^{\prime}$, then there exists a sequence $\left(x_{n}\right)$ which is transformed by $f(M)$ into a sequence converging to zero, and such that the sequences obtained by omitting and by adjoining an element are transformed by $f(M)$ into sequences which do not converge to zero.

As in $\S 4$, write $p=1 / \rho-1$. Since $\rho$ is in $C^{\prime}, \mathbf{R}(p) \geqq 1$; assuming first that $\rho \neq \frac{1}{2}, \frac{1}{3}, \frac{1}{4}, \cdots$, we find that $p \neq 1,2,3, \cdots$. Define*

$$
x_{n}=\frac{\Gamma(n)}{\Gamma(n-p)} ;
$$

then denoting the transformed sequence by $\left(y_{n}\right)$,

so that

$$
y_{n}=\frac{1}{n \Gamma(1-p)},
$$

$$
\lim _{n=\infty} y_{n}=0 \text {. }
$$

If we apply $f(M)$ to the sequence $x_{2}, x_{3}, x_{4}, \cdots$, we have

$$
\eta_{n}=\frac{1}{n p^{2} \Gamma(-p)}+\frac{1}{p} \frac{\Gamma(n)}{\Gamma(n-p+1)} ;
$$

\footnotetext{
${ }^{*}$ The sequences $\left(x_{n}\right),\left(y_{n}\right)$-here are exactly the sequences $\left(y_{n}\right),\left(x_{n}\right)$ respectively of $\S 4$.
} 
and applying $f(M)$ to the sequence $0, x_{1}, x_{2}, \cdots$,

It is evident that

$$
\eta_{n}^{\prime}=\frac{1}{n \Gamma(1-p)}-\frac{1}{p} \frac{\Gamma(n-1)}{n \Gamma(n-p-1)} .
$$

$$
\lim _{n=\infty} \eta_{n}=\infty
$$

for $\mathbf{R}(p)>1$, that is, for $\rho$ inside $C^{\prime}$; for $\rho$ on the boundary of $C^{\prime}, \mathbf{R}(p)=1$, and

$$
\lim _{n=\infty}\left|\eta_{n}\right|=\frac{1}{|p|} \neq 0
$$

We see likewise, by writing

that

$$
\frac{\Gamma(n-1)}{n \Gamma(n-p-1)}=\frac{n-2}{n} \frac{\Gamma(n-2)}{\Gamma(n-p-1)}
$$

$$
\lim _{n=\infty} \eta_{n}^{\prime}=\infty
$$

unless $\rho$ is on the boundary of $C^{\prime}$, and in this exceptional case,

$$
\lim _{n=\infty}\left|\eta_{n}^{\prime}\right|=\frac{1}{|p|} \neq 0 \text {. }
$$

We have excluded the cases $\rho=\frac{1}{2}, \frac{1}{3}, \cdots$. If $\rho$ has any one of these values some of the earlier elements of the sequence $\left(x_{n}\right)$ defined above become meaningless, since they involve in the denominators gamma functions of zero or negative integers; if, however, we replace each such meaningless element by zero, the preceding proof holds without alteration.

\section{Conclusion}

The class of analytically regular definitions considered in the preceding pages obviously includes a wide variety of definitions given by linear transformations. It does not, however, include all such definitions; for instance it fails to cover the logarithmic definitions of Riesz, ${ }^{*}$ which are not permutable with $M$.

The consistency of all analytically regular definitions and the simplicity of the criteria for the equivalence and the relative generality of any two of them introduce a considerable degree of system into the study of such divergent series as may be successfully treated by this particular class of definitions. It is all the more important, therefore, to point out some desiderata in the theory. In the first place, some substitute for Theorem $\mathrm{V}$, involving only

* Paris Comptes Rendus, vol. 149 (1909), p. 18. 
real variables and conditions appropriate to real variables, is desirable, in order to remove the irksome requirement of analyticity in C. Again, consistency breaks down if the notion of limit be extended to include real onesigned infinity; for instance, the sequence $0,1,2,3, \cdots$ is evaluated by the analytically regular definition* $2 M-E$ to the value 0 .

It is probable that a natural generalization exists of Cesàro's results $\dagger$ on the Cauchy-product of summable series, and of the theorem of Frobenius on the behavior of a real power-series summable at an end of its interval of convergence.

Finally, the general results of the paper should admit of extension to the case of the limit of a continuous variable. The foundation for this extension exists in a paper by Silverman§ establishing conditions for regularity similar to (1). The further theorems analogous to those of the present article will be treated in a future paper.

* Other examples of the same type are contained incidentally in the proof of Theorem XIV.

$\dagger$ Bromwich, An Introduction to the Theory of Infinite Series, p. 315.

$\ddagger$ Bromwich, l. c., p. 312 .

§ These Transactions, vol. 17 (1916), p. 284; see also Bulletin of the American Mathematical Society, vol. 22 (1916), p. 459. 